\title{
Procesado mediante una ruta pulvimetalúrgica de materiales compuestos Mg-10Ti (\% vol)
}

\author{
P. Pérez", G. Garcés ${ }^{* *}$ y P. Adeva*
}

\begin{abstract}
Resumen El presente estudio trata sobre la caracterización mecánica, en el intervalo de temperaturas comprendido entre 25 y $300^{\circ} \mathrm{C}$, del material compuesto de matriz magnesio $\mathrm{Mg}-10 \mathrm{Ti}$ (\% vol.) obtenido a través de una ruta pulvimetalúrgica que incluye la mezcla de los polvos de magnesio y titanio, compactación en frío y extrusión a $400{ }^{\circ} \mathrm{C}$. El material muestra una elevada resistencia $(218 \mathrm{MPa})$ y alargamiento $(4,7 \%)$ a temperatura ambiente, valores superiores a los de la mayoría de materiales compuestos de matriz magnesio reforzados con un material cerámico. La reducción en las propiedades mecánicas del material se produce de un modo gradual a partir de $100^{\circ} \mathrm{C}$, lo que indica el efecto beneficioso del refuerzo de titanio.
\end{abstract}

Palabras clave Pulvimetalurgia. Propiedades mecánicas. Material compuesto. Magnesio. Refuerzo metálico.

\section{Powder metallurgy processing of $\mathrm{Mg}-10 \mathrm{Ti}$ (\% vol.) composites}

\begin{abstract}
The present study deals with the mechanical characterization at temperatures between 25 and $300{ }^{\circ} \mathrm{C}$ of a magnesium matrix composite reinforced with a $10 \%$ volume fraction of titanium particles. The $\mathrm{Mg}-10 \mathrm{Ti}$ ( vol.\%) composite was processed by a powder metallurgy involving mixing of powders, cold pressing and extrusion at $400{ }^{\circ} \mathrm{C}$. The composite shows high strength $(218 \mathrm{MPa})$ and some elongation $(4,7 \%)$ at room temperature, higher than those reported for most of magnesium matrix composites. The gradual decrease in the mechanical properties of the composite at temperatures above $100{ }^{\circ} \mathrm{C}$ indicates the beneficial effect of titanium reinforcement.
\end{abstract}

Keywords Powder metallurgy. Mechanical properties. Composite. Magnesium. Metallic reinforcement.

\section{INTRODUCCIÓN}

El interés en las aleaciones de magnesio en aplicaciones estructurales se debe a que es el metal estructural que presenta una menor densidad y una elevada resistencia específica. Sin embargo, tanto su escasa resistencia frente a la corrosión como sus bajas propiedades mecánicas han limitado su uso en aplicaciones muy específicas. Para poder ampliar el campo de posibles aplicaciones estructurales sería necesario mejorar ambas propiedades.

Las propiedades mecánicas del magnesio se pueden modificar y mejorar mediante un control de la microestructura a través de una elección adecuada del procesado termomecánico del material. Sin embargo, en el caso del magnesio y sus aleaciones este aumento de propiedades queda restringido a temperaturas inferiores a $200^{\circ} \mathrm{C}$. Este comportamiento está íntimamente relacionado con los mecanismos de deformación del magnesio. A bajas temperaturas, sólo se activa el sistema de deslizamiento basal, mientras que al aumentar la temperatura también se activan el deslizamiento prismático y el piramidal. Por tanto, el control de la composición química y de la microestructura de la aleación es sólo efectivo a bajas temperaturas. En este punto, se puede considerar el diseño de materiales compuestos de matriz magnesio como una alternativa para obtener materiales con elevadas propiedades mecánicas y alta resistencia a la fluencia. Los refuerzos que generalmente se han empleado son cerámicos $\left(\mathrm{Al}_{2} \mathrm{O}_{3}, \mathrm{Y}_{2} \mathrm{O}_{3}, \mathrm{TiB}_{2}, \mathrm{Mg}_{2} \mathrm{Si}, \mathrm{SiC}\right.$,

(*) Centro Nacional de Investigaciones Metalúrgicas (CENIM), Avda. Gregorio del Amo, 8, 28040 Madrid, España.

$(* *)$ Max Planck Institute for Metals Research, Heisenbergstrasse 3, D-70569 Sttutgart, Germany. 
$\mathrm{B}_{4} \mathrm{C}$, etc...). Éstos, producen un aumento escaso del límite elástico que suele ir acompañado por una disminución apreciable del alargamiento a rotura $^{[1-3]}$. Este comportamiento se ha asociado a una intercara matriz-refuerzo débil, muchas veces generada por una reacción química entre el refuerzo y la matriz en el procesado, por porosidad o por la generación de daño durante la deformación.

El empleo de una dispersión de un metal insoluble en el magnesio podría reforzar la matriz de un modo más efectivo, por varias razones: i) La menor diferencia entre los coeficientes de expansión térmica entre el refuerzo y la matriz disminuiría el riesgo de que se forme porosidad o daño en la intercara matriz-refuerzo; ii) la inmiscibilidad del metal reforzante en la matriz excluye el riesgo de la formación de fases que debiliten la intercara matriz-refuerzo; y iii) el refuerzo podría deformarse plásticamente en zonas locales sometidas a mayores esfuerzos, como pueden ser los extremos de grieta.

Basado en este análisis, el presente estudio trata de analizar la influencia de la adición de un $10 \%$ en volumen de un refuerzo de titanio en las propiedades mecánicas del magnesio puro

\section{MATERIALES Y PROCEDIMIENTO EXPERIMEN- TAL}

El material compuesto $\mathrm{Mg}$-10Ti (\% vol.) se obtuvo mediante una vía pulvimetalúrgica. En un molino de bolas, se mezclaron polvos comerciales de magnesio puro con un tamaño de partícula inferior a $45 \mu \mathrm{m}$ con la fracción correspondiente de polvos de titanio con un tamaño inferior a $25 \mu \mathrm{m}$. La mezcla se realizó al aire y la relación entre la masa de las bolas y la masa de los polvos fue de 4 . Se emplearon dos condiciones: i) 120 r.p.m. durante $1 \mathrm{~h}$ y ii) 70 r.p.m. durante 5 h. La mezcla fue posteriormente compactada uniaxialmente en frío hasta alcanzar una densificación cercana al $90 \%$. Finalmente, el material fue extruido a $400{ }^{\circ} \mathrm{C}$ con una relación de extrusión de 18/1.

La caracterización microestructural del material se efectuó mediante microscopía óptica $(\mathrm{MO})$ y electrónica de barrido (MEB). Las propiedades mecánicas del material se determinaron mediante ensayos de tracción entre temperatura ambiente y $300{ }^{\circ} \mathrm{C}$, a una velocidad de deformación de $10^{-4} \mathrm{~s}^{-1}$. De las barras se mecanizaron probetas cilíndricas de $3 \mathrm{~mm}$ de diámetro y $7 \mathrm{~mm}$ de longitud de ensayo. Igualmente, a 100,200 y $300{ }^{\circ} \mathrm{C}$ se realizaron ensayos con saltos en la velocidad de defor- mación, a fin de determinar la relación entre ésta y la tensión.

\section{RESULTADOS}

La figura 1 muestra la microestructura del material extrudido en la condición de mezcla $1 \mathrm{~h}$ a 120 r.p.m. Se puede comprobar que las condiciones de mezcla de los polvos de magnesio y titanio producen una distribución homogénea de las partículas del refuerzo en la matriz de magnesio. Las partículas originales de magnesio están muy alargadas en la dirección de extrusión y se puede, igualmente, observar el flujo de las partículas de magnesio alrededor del refuerzo. Coincidiendo con las fronteras de las partículas originales de magnesio se encuentran pequeñas partículas de óxido, que se generan por la rotura de las capas de óxido presentes en la superficie de las partículas de polvo originales. Además, del refuerzo de titanio y de las partículas de óxido, se encontró una pequeña fracción en volumen de partículas de otra naturaleza. Mediante microanálisis por dispersión de energía (EDX) se comprobó que estas partículas eran $\mathrm{CaO}$ (con un tamaño similar al refuerzo) y hierro (mucho más dispersa y mucho más pequeñas que las partículas de titanio). $\mathrm{El} \mathrm{CaO}$, probablemente, proviene de desoxidantes utilizados durante el procesado del material a fin de evitar la oxidación del polvo de magnesio. El hierro, podría derivar de los tarros y bolas empleados en la etapa de mezcla. En cualquier caso, no se observó ni la presencia de porosidad ni la formación de nuevas fases por reacción entre la matriz y el refuerzo. El material compuesto

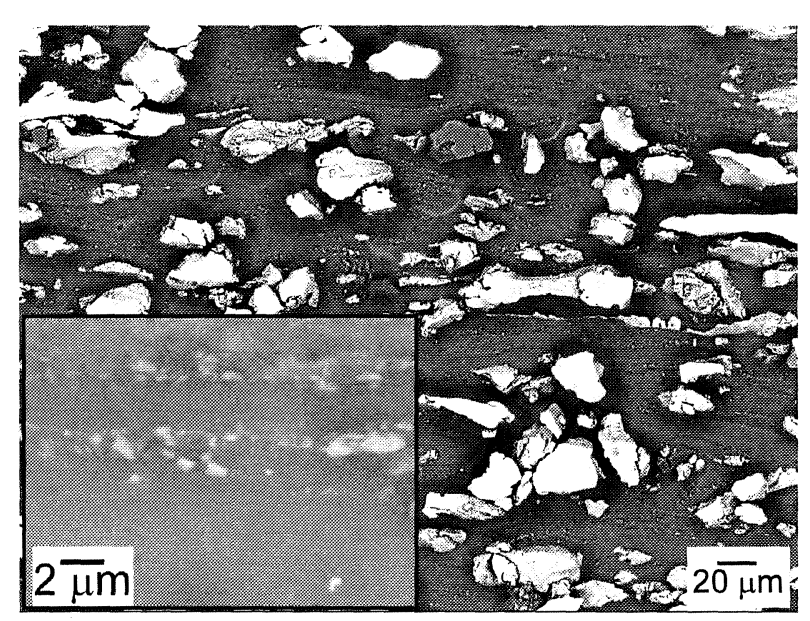

Figura 1. Microestructura del material compuesto mezclado 1 h a 120 r.p.m.

Figure 1. Microstructure of the composite mixed for $1 \mathrm{~h}$ at 120 r.p.m. 
en el que la mezcla se realizó durante 5 h a 70 r.p.m. presenta una microestructura muy parecida, si bien el refuerzo se encuentra algo más aglomerado. Independientemente de las condiciones de mezcla, el tamaño de grano de la matriz de magnesio es muy heterogéneo y queda determinado, en muchos casos, por el tamaño de la partícula original de polvo y el grado de deformación experimentado por dicha partícula en la extrusión, como se observa en la figura 2. Un hecho importante a destacar, es el crecimiento de grano durante la extrusión, que en muchos casos puede llegar a traspasar las partículas originales de magnesio.

La figura 3 muestra las curvas tensión real-deformación real del material compuesto, en la condición de 1 h, 120 r.p.m., ensayado entre 25 y $300^{\circ} \mathrm{C}$. Las curvas $\sigma-\varepsilon$ se caracterizan por el endurecimiento en el periodo inicial de la deformación, que es seguido por un periodo estacionario. Se observan altos valores de resistencia a temperatura ambiente (218 $\mathrm{MPa})$ que tienden a disminuir de un modo progresivo a medida que aumenta la temperatura de ensayo, de manera que a $300^{\circ} \mathrm{C}$ la resistencia máxima es de $50 \mathrm{MPa}$. El máximo alargamiento, $11,5 \%$, correspondió al material deformado a $100{ }^{\circ} \mathrm{C}$, mientras que no superó el $5 \%$ en el resto de temperaturas. Curiosamente, el menor alargamiento, $3,4 \%$, se midió a $300{ }^{\circ} \mathrm{C}$. No se observó que las condiciones de mezcla influyan, de un modo apreciable, en los valores de las propiedades mecánicas del material compuesto, como se puede comprobar en la tabla I.

La figura 4 representa la velocidad de deformación frente a la tensión en el estado estacionario, a

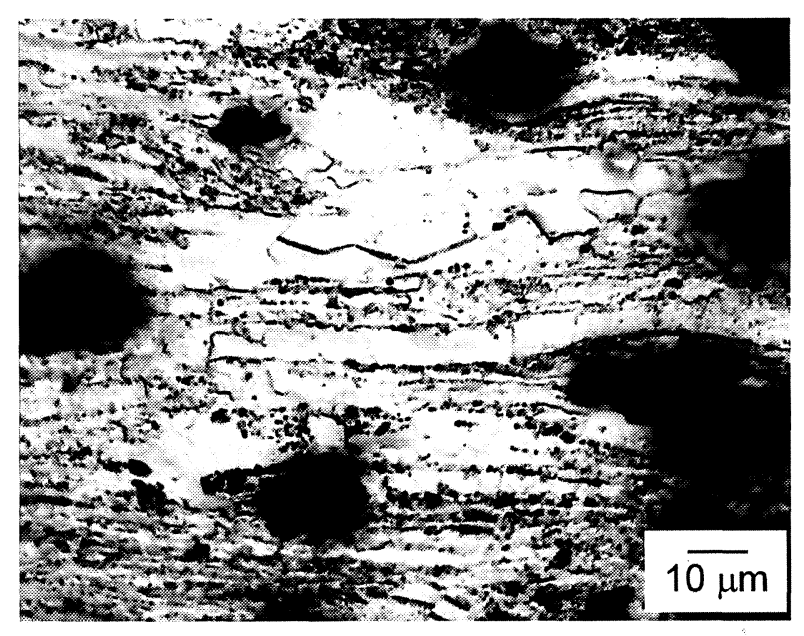

Figura 2. Microestructura de la matriz de magnesio en el material compuesto mezclado 5 h a 70 r.p.m.

Figure 2. Magnesium matrix microstructure of the composite mixed for $5 h$ at 70 r.p.m.

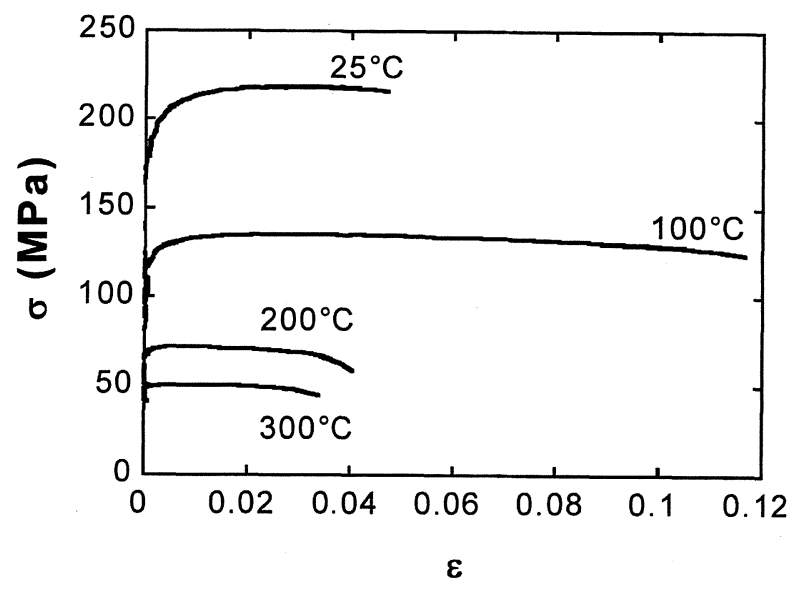

Figura 3. Curvas tensión real-deformación real entre 25 y $300{ }^{\circ} \mathrm{C}$ del material compuesto mezclado durante $1 \mathrm{~h} \mathrm{a}$ 120 r.p.m.

Figure 3. Tensile true stress-true strain curves between 25 and $300{ }^{\circ} \mathrm{C}$ for the composite mixed at 120 r.p.m. for $1 \mathrm{~h}$.

Tabla I. Límite elástico, resistencia máxima, alargamiento y exponente de la tensión para los dos materiales compuestos procesados con las distintas condiciones de mezcla Table I. Yield stress, maximum strength, elongation, and stress exponent values for the two Mg-Ti composites processed under different mixing conditions

\begin{tabular}{|c|c|c|c|c|c|c|c|c|}
\hline \multirow{2}{*}{$\begin{array}{c}\text { Temperature } \\
{ }^{\circ} \mathrm{C}\end{array}$} & \multicolumn{4}{|c|}{ Mg-10Ti 1 h 120 r.p.m. } & \multicolumn{4}{|c|}{ Mg-10Ti 5 h 70 r.p.m. } \\
\hline & $\sigma_{0}$ & $\mathbf{R}_{\mathrm{m}}$ & $\varepsilon$ & $\mathrm{n}$ & $\sigma_{0}$ & $\mathbf{R}_{\mathrm{m}}$ & $\varepsilon$ & $\mathbf{n}$ \\
\hline 25 & 197 & 219 & 4,8 & & 185 & 201 & 4,7 & \\
\hline 100 & 124 & 135 & 11,7 & 13,8 & 127 & 138 & 9,5 & 11,2 \\
\hline 200 & 70 & 72 & 4 & 11,9 & 69 & 72 & 4,3 & 12,7 \\
\hline 300 & 49 & 49 & 3 & 14,5 & 46 & 51 & 4 & 15,7 \\
\hline
\end{tabular}

100,200 y $300{ }^{\circ} \mathrm{C}$ para el material compuesto mezclado 5 h a 170 r.p.m. A cada temperatura, se han ajustado los puntos de acuerdo con la ecuación potencial de la fluencia

$$
\dot{\varepsilon}=k \sigma^{n_{a p}} e^{\left(-Q_{a p} / R T\right)}
$$

donde, $\mathrm{k}$ es una constante, $\mathrm{n}_{\mathrm{ap}}$, el exponente aparente de la tensión, $\sigma$, la tensión en el estado estacionario, $Q_{a p}$, la energía de activación para la fluencia, $\mathrm{T}$, la temperatura absoluta y $\mathrm{R}$, la constante universal de los gases. Se puede comprobar que el exponente de la tensión, $n$, alcanza valores superiores a 10 , a las tres temperaturas. Los valores calculados en el caso del material compuesto mezclado durante $5 \mathrm{~h}$ a 70 r.p.m. fueron muy similares, 


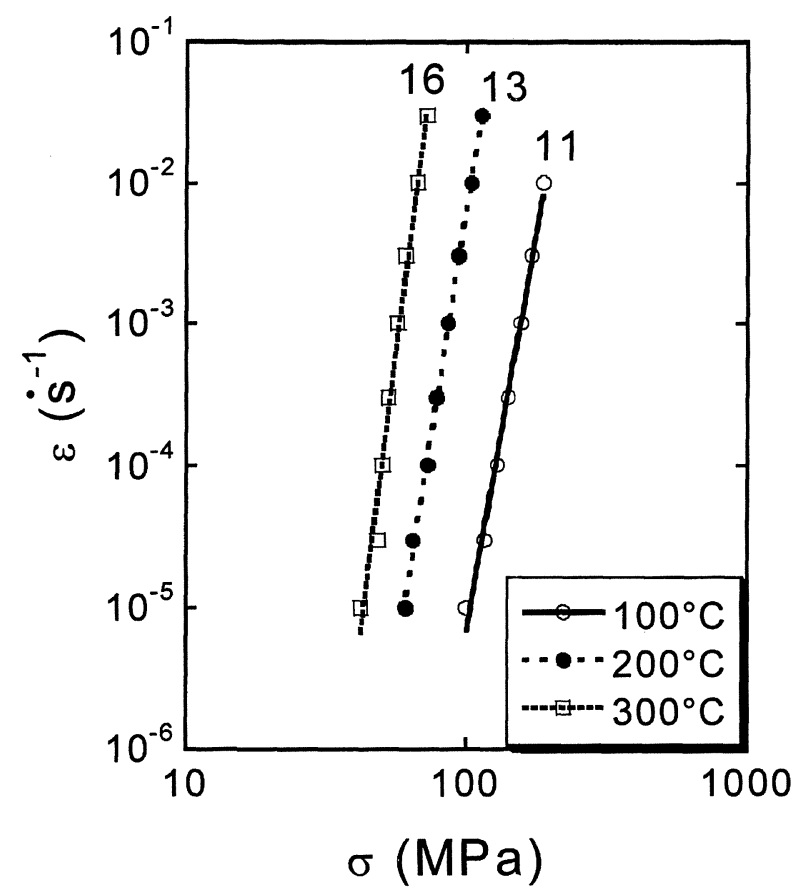

Figura 4. Gráfico de la velocidad de deformación-tensión en el estado estacionario entre 100 y $300^{\circ} \mathrm{C}$ del material compuesto mezclado durante 5 h a 70 r.p.m.

Figure 3. Strain rate-steady stress plot between 100 and 300 ${ }^{\circ} \mathrm{C}$ for the composite mixed at 70 r.p.m. for $5 \mathrm{~h}$.

si bien se obtuvieron valores crecientes de $\mathrm{n}$ al aumentar la temperatura (Tabla I). A partir de estos datos se puede calcular la energía de activación para la fluencia, que varía entre $121-127 \mathrm{~kJ} / \mathrm{mol}$ para la aleación mezclada $1 \mathrm{~h}$ a 120 r.p.m. y $111-128 \mathrm{~kJ} / \mathrm{mol}$ en el caso del material mezclado $5 \mathrm{~h}$ a 70 r.p.m. Estos valores próximos al valor de la autodifusión del magnesio, $135 \mathrm{~kJ} / \mathrm{mol}$, indican que el mecanismo de deformación entre 100 y $300^{\circ} \mathrm{C}$ está controlado por dicho proceso.

Las superficies de fractura muestran en ambas aleaciones una fractura dúctil, con gran número de microcavidades en la matriz de magnesio (Fig. 5). En esta misma fractura, se puede observar, igualmente, que las partículas de $\mathrm{CaO}$ se fracturan durante la deformación (ver Fig. 5). La observación de la sección metalográfica de la fractura indica que la grieta que produce la fractura se propaga, fundamentalmente, en la matriz de magnesio bordeando las partículas de titanio.

\section{DISCUSIÓN}

Los altos valores de las propiedades mecánicas obtenidos en este material, cuya matriz es magnesio puro, junto a la distribución homogénea del re-

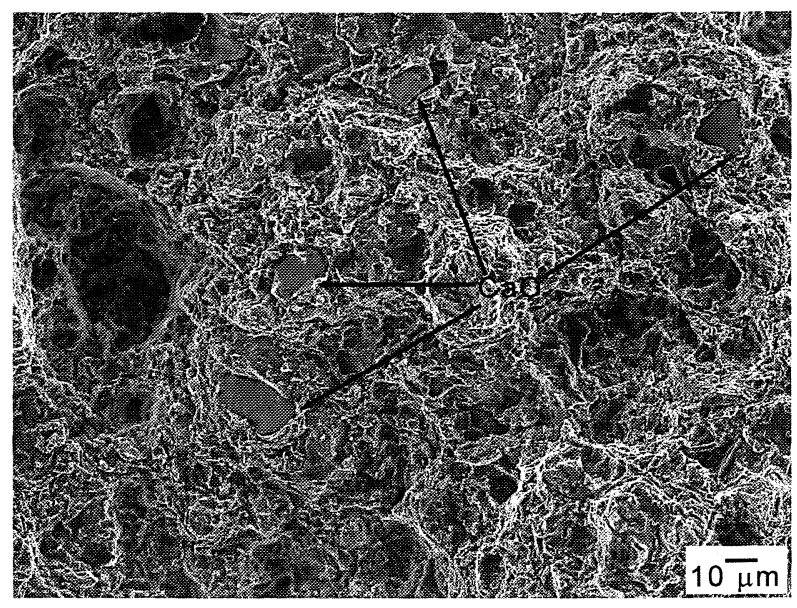

Figura 5. Superficie de fractura a temperatura ambiente del material compuesto $\mathrm{Mg}$-10Ti mezclado 1 h a 120 r.p.m.

Figure 5. Fracture surface at room temperature of the Mg-10Ti composite mixed at 120 r.p.m. for $1 \mathrm{~h}$.

fuerzo de titanio indican que las dos condiciones de mezcla son idóneas para procesar el material. Puesto que las propiedades mecánicas son muy similares en ambos materiales, se puede concluir que las condiciones de mezcla de los polvos de magnesio y titanio empleados producen una microestructura muy similar en ambas aleaciones. La microestructura de la matriz de magnesio es muy heterogénea. Esta heterogeneidad tiene dos causas:

- El polvo original de magnesio, $<45 \mu \mathrm{m}$, presenta una distribución de tamaños, de tal modo que tras la extrusión las partículas deformadas pueden presentar anchuras muy diferentes en función del diámetro original y el grado de deformación que experimente durante la extrusión.

- La mayor deformación que, generalmente, experimentan las partículas de magnesio que interaccionan con las partículas de titanio. Esto se puede ver claramente en la gran distorsión que experimenta el flujo de las partículas deformadas de magnesio en las proximidades del refuerzo de titanio. En estas regiones, el grado de deformación es, generalmente, mayor que en las regiones libres de refuerzo.

Durante la extrusión, se destruye la capa de óxido que recubre las partículas de magnesio y titanio. Ello, produce una dispersión de pequeñas partículas de óxido que se sitúan en las fronteras de las partículas originales de polvo. Estas partículas son las que anclan el grano, e impiden el 
crecimiento de grano de la matriz de magnesio durante la extrusión a $400^{\circ} \mathrm{C}$. Así, una gran parte del tamaño de los granos de magnesio queda delimitado por el diámetro de la partícula deformada. A esta temperatura, sin embargo, esta franja discontinua de óxidos no es completamente efectiva y el crecimiento del grano puede abarcar partículas vecinas. Por tanto, las partículas de óxido se pueden situar tanto en frontera de grano como en el interior. Esta disposición de las partículas afecta igualmente a las propiedades mecánicas del material.

Los elevados valores del exponente de la tensión, $n$, denotan la gran contribución del refuerzo y de las partículas de óxido a endurecer el material. Estos valores son mucho mayores que los calculados en otros materiales compuestos de magnesio puro como matriz ${ }^{[3-5]}$. De acuerdo con los estudios de Milička et al. una parte importante del refuerzo se debe a la interacción de las dislocaciones con las pequeñas partículas de óxido ${ }^{[6]}$. Estos autores encontraron que la dispersión de un 0,11 \% en masa de partículas de $\mathrm{MgO}$ aumenta de un modo considerable el valor del exponente de la tensión, $n$, entre 127 y $287^{\circ} \mathrm{C}$, en comparación con el magnesio puro $(n \approx 5)^{[7]}$, hasta alcanzar valores de 8 a 10 . En el presente estudio, el aumento adicional de $n$ debe asociarse a la contribución del refuerzo de titanio. Puesto que el refuerzo de titanio es relativamente grande, el mecanismo que debería actuar sería la transferencia de carga de la matriz al refuerzo. Ambas contribuciones causan un gran endurecimiento que se traduce en las elevadas propiedades mecánicas que muestra el material en todo el intervalo de temperaturas, manteniendo una cierta ductilidad, comparadas con las propiedades del magnesio puro. La escasa ductilidad a 200 y $300^{\circ} \mathrm{C}$, junto a los más elevados valores de $\mathrm{n}$, sugieren que la interacción entre el refuerzo y la matriz debe ser la máxima que puede soportar el material. A ello, contribuiría la fuerte intercara entre el refuerzo de titanio y la matriz. Una mejora adicional de las propiedades mecánicas a bajas temperaturas se podría conseguir evitando el crecimiento del grano durante la etapa de consolidación, bajando la temperatura de extrusión.

\section{CONCLUSIONES}

- Se pueden procesar, mediante una vía pulvimetalúrgica, materiales compuestos de matriz magnesio reforzados con un $10 \%$ en volumen de partículas de titanio. Las condiciones de mezcla empleadas en el presente estudio son adecuadas para obtener una microestructura homogénea.

- El material compuesto Mg-10Ti, procesado por esta vía pulvimetalúrgica, muestra unas propiedades mecánicas muy elevadas en todo el intervalo de temperaturas.

- Los altos valores del exponente de la tensión indican la gran contribución del refuerzo en el endurecimiento del material.

- Se puede lograr un aumento adicional de las propiedades del material compuesto refinando la microestructura de la matriz.

\section{Agradecimientos}

P. Pérez agradece a la CICYT la ayuda dentro del programa Ramón y Cajal. Igualmente, se agradece la financiación de la CAM a través del proyecto CAM 07N 0075-2002.

\section{REFERENCIAS}

[1] M. Mabuchi, K. Kubota y K. Higashi, Scripta Metall. Mater. 33 (1995) 331-334.

[2] B.Q. Han y D.C. Dunand, Mater. Sci. Eng. A277 (2000) 297-304.

[3] H. Ferkel y B.L. Mordike, Mater. Sci. Eng. A298 (2001) 193-199.

[4] V. SkleničKa, M. Pahutová, K. Kucha?ová, M. Svoboda y T.G. LANGDON, Metall. Mater. Trans. 33A (2002) 883 889.

[5] B.Q. Han y D.C. Dunand, Mater. Sci. Eng. A319-321 (2001) 741-745.

[6] K. MiličKa, J. ČadeK y P. RYš, Acta. Metall. 18 (1970) 1071-1082.

[7] S.S. Vagarali y T.G. Langdon, Acta Metall. 29 (1981) 1969-1982. 